\title{
Effects in extinction, with and without free choice, of degree of experience with stimuli associated with both partial and continuous reinforcement'
}

\author{
MICHAEL DIVAK2 AHO ROGERS ELLIOTT
}

DARTMOUTH COLLEGE

The effect of two degrees of training of rats to both arms of a T-maze, one associated with partial reinforcement and the other with continuous, were investigated. Animals were permitted free choice of arms in extinction trials, and extra training did not diminish the strong preference for CRF. In a third, no-choice group, animals were extinguished in one arm or the other, but not both: extinction took as long to $\mathrm{CRF}$ - as it did to PR-associated stimuli.

A greater resistance to extinction is commonly found among animals trained on partial reinforcement (PR) schedules when they are compared, with others trained on continuous reinforcement (CRF): this result constitutes the partial reinforcement effect (PRE). The PRE does not appear, however, if the same animal is trained on both schedules and then tested with responses to both the PR- and CRF-associated stimuli freely and simultaneously available.

Such a reversed PRE in a single group (within-S) design where the choice stimuli are arms of a maze is very strong (Mason, 1957; D'Amato, Lachman, \& Kivy, 1958), and Lawrence \& Festinger (1962) attempted to account for it by supposing that the "extra attractions" presumably developed through experience with PR are weak relative to the attractions associated with CRF-associated stimuli, at least early in extinction.

Furthermore, the attractions which develop from experience with deterrents take some time to develop, so that the extent of such experience is critical in testing for such attractions. The point has been made in another context by Mandler (1964), who speculated that prolonged overtraining on PR schedules might lead to preference for PR- over CRF-associated stimuli. The argument seems to be that the arousal and uncertainty that are large and disruptive early in PR training eventually, after counterconditioning and habituation, reduce to levels that are mild enough to be attractive.

Of the two studies referred to, Mason (1957) trained his animals extensively, but his acquisition task was relatively complex; and D'Amato et al (1958) did not train very extensively, even though they used a very simple training procedure. We employed a form of the simpler task, with two degrees of training, in effect adding an overtraining group to the design used by $D^{\prime}$ Amato et al to check for possible increases in the strength of "extra attractions" with experience with PR. We also followed their astute suggestion, in a third group, of training each animal on both PR and CRF, and then testing half of them on PR extinction and the other half on CRF extinction. Such a procedure would seem to meet the objections made by Lawrence \& Festinger (1962), because the attractions developing with PR training would not be in any competition with expectancies for food aroused by CRF stimuli, and hence should not be masked.

\section{Method}

Subjects. The Ss were 26 naive male Holtzman rats 90-100 days old, maintained at $80-85 \%$ free-feeding weight during experimentation.

Apparatus. The T-maze used had a stem 9 in. long and 4 in. wide, and a movable masonite door could be inserted and removed in slots placed just before the juncture of the stem (which served as the start box) with the crossing arms. The overall length of the crossing arms was 22 in., and the width 4 in. The choice point area just beyond the stem used 4 in. of the 22 in., leaving 9 in. for each goal area. These goal areas were isolatable from the choice point area by masonite doors like those described above. At the end of each goal area was a food trough, in the base of which was embedded a 5-gm pellet, visible behind a screen, as control for olfactory cues. The 45-mg Noyes pellets used as reinforcers were manually placed in the troughs each time one was scheduled, with simultaneous dropping of another pellet into the other trough to control for sound cues.

Procedure. Animals were on deprivation regime for 10 to 18 days prior to the familiarization procedures: eating pellets randomly placed in the maze, and tabletop gentling.

All three groups of rats received the same within-S training on the first four days. Day 1 training consisted of 50 forced, massed trials to one arm for one condition; e.g. VR2. On Day 2 the other arm was used for 50 forced, massed trials of the other schedule; e.g., CRF. On Day 3, there were 52 trials, 26 to continuous and 26 to partial; and again on Day 4,52 trials, 26 trials to continuous and 26 to partial. As can be seen, the number of trials to each arm was equated and the number of reinforcements was not. The order of presentation of the two schedules was randomly determined. On any trial the $S$ remained $10 \mathrm{sec}$. in 
Table 1. Number of partial and complete choices, and distribution of the first 10 choices to PR and CRF arms, for the groups with less and more training; and data on trials to extinction for the within-between group.

\begin{tabular}{lcc} 
& To PR Arm & To CRF Arm \\
\hline Less Training (N=8): & & \\
\% First Ten Choices & 42.5 & $57.5^{* * *}$ \\
Total Number, All Choices & 24.6 & 33.4 \\
Total Number, Complete Choices & 15.4 & $24.9 * * *$ \\
More Training (N=8): & & \\
\% First Ten Choices & 33.8 & $66.2^{*}$ \\
Total Number, All Choices & 32.8 & $50.1^{* *}$ \\
Total Number, Complete Choices & 21.0 & $39.2^{* * *}$ \\
Within-Between (N=10): & & \\
Total Number, All Trials & 101.4 & 86.4 \\
Total Number, Complete Trials & 35.2 & 35.0 \\
\hline
\end{tabular}

$* * * 0.01$, one-tailed t-test

** $p .025$, one-tailed t-test

* p.05, one-tailed t-test

start box with all doors closed. The start door and one programmed door were opened simultaneously and shut when $\mathbf{S}$ was in goal box. Fifteen sec. after pellet eating commenced $S$ was returned to start box. The animal remained in goal box for 15 sec. on trials when no pellets were given. The association of position of arm with condition of reinforcement was balanced across animals during training.

The eight animals in the group with more training (MT) differed from the eight animals in the group with less (LT) in that, instead of being tested on Day 5, they were given eight additional days of training (416) trials like that given on Days 3 and 4. Testing for groups MT and LT was a within-S procedure; i.e., both PR and CRF arms were made available for the first time together by opening all three doors simultaneously. When the animal looked into the food trough, the door of the arm into which he had entered was closed and the trial considered complete. If no trough was selected in $30 \mathrm{sec}$., the doors were shut again, and the animal was placed in start again. Extinction was considered complete if $\mathrm{S}$ did not leave the start box for five consecutive trials. Testing was arbitrarily stopped at three hours for the MT group; maximum time to extinction in the LT group was two hours.

The third group, designated Within-Between (WB), was given two days of training more than the LT animals, so that their first test day was Day 7, at which time half (five animals) began extinction trials to the PR arm, and the other half to the CRF arm; i.e., one door was always closed during extinction trials, and these arrangements were visible to the animal from his starting position when the start door was lifted. The performance measures for the LT and MT groups were number of choices of an arm in extinction, a choice being scored with any part of the animal's body entered in the arm; number of complete trials, that subset of choices in which the animal moved all the way to the food trough; and percent of the first 10 choices to either arm. For the WB animals, the scores were the number of trials during extinction, and the number in subclass of trials in which they went completely to the food trough. They were run "blind" by the E.

\section{Results and Discussion}

The major results appear in Table 1. Comparing the LT with the MT group, it is clear that the extra training of the MT group did not result in any diminution of preference for the CRF arm during the test trials in extinction. In each group, only one animal showed a preference for the PR arm in terms of proportion of the first 10 trials, or in terms of the total number of complete choices; in terms of the total number of choices of any degree, only two animals in the LT group and one in the MT group preferred the PR arm. The degree of preference for CRF was, against our expectation, slightly greater in the $M T$ group than in the LT group, though this difference in degree of preference was by no means significant for any measure. It can be said with confidence that extra training, at least to the degree involved here, did not make "extra attraction" for the PR arm more obvious in the situation of simultaneous choice between PR- and CRF-associated stimuli.

When the animals in the WB group, not given any such choice, were extinguished, there was no significant difference by either of the two measures employed in the degree to which PR- or CRF-associated stimuli affected resistance to extinction, though the total-trial-to-extinction measure was slightly in the direction one would expect by predicting that the usual PRE would occur. Five animals in a group are not many, and it is clear that this design ought to be more extensively investigated, since it does seem to offer a critical evaluation of the validity of the arguments advanced by Lawrence \& Festinger (1962) against results like those reported by Mason (1957) and D'Amato et al (1958).

\section{References}

D'Amato, M. R., Lachman, R., \& Kivy, P. Secondary reinforcement as affected by reward schedule and the testing situation. $J$. comp. physiol. Psychol., 1958, 51, 737-741.

Lawrence, D., \& Festinger, L. Deterrents and reinforcement. Stanford: Stanford University Press, 1962.

Mandler, G. The interruption of behavior. In M. R. Jones (Ed.), Nebraska symposium on motivation. Lincoln: University of Nebraska Press, 1964, 163-219.

Mason, D. J. The relation of secondary reinforcement to partial reinforcement. J. comp. physiol. Psychol., 1957, 50, 264-268.

\section{Notes}

1. We thank H. Buchtel, J. Lanzetta, R. Leaton, R. Musty, and T. Tighe for their many suggestions. The study was carried out by the first author under the direction of the second in partial satisfaction of the requirements for the senior undergraduate courses in independent research.

2. Now at Princeton University. 